\title{
Enhancing a Simple MODIS Cloud Mask Algorithm for the Landsat Data Continuity Mission
}

by

Michael J. Wilson ${ }^{1,2}$ and Lazaros Oreopoulos ${ }^{2}$

1. Joint Center for Earth Systems Technology, University of Maryland Baltimore County, Baltimore, MD 21250

2. Climate and Radiation Laboratory, NASA-Goddard Space Flight Center, Code 613, Greenbelt, MD 20771

Submitted to

IEEE Transactions on Geoscience and Remote Sensing

September 2011

Corresponding author address:

Lazaros Oreopoulos

NASA-GSFC

Code 613.2

Greenbelt, MD 20771

USA

Lazaros.Oreopoulos@inasa.gov 


\section{Popular Summary}

The presence of clouds in images acquired by the Landsat series of satellites is usually an undesirable, but generally unavoidable fact. With the emphasis of the program being on land imaging, the suspended liquid/ice particles of which clouds are made of fully or partially obscure the desired observational target. Knowing the amount and location of clouds in a Landsat scene is therefore valuable information for scene selection, for making clear-sky composites from multiple scenes, and for scheduling future acquisitions.

The two instruments in the upcoming Landsat Data Continuity Mission (LDCM) will include new channels that will enhance our ability to detect high clouds which are often also thin in the sense that a large fraction of solar radiation can pass through them. This work studies the potential impact of these new channels on enhancing LDCM's cloud detection capabilities compared to previous Landsat missions. We revisit a previously published scheme for cloud detection and add new tests to capture more of the thin clouds that are harder to detect with the more limited arsenal channels. Since there are no Landsat data yet that include the new LDCM channels, we resort to data from another instrument, MODIS, which has these bands, as well as the other bands of LDCM, to test the capabilities of our new algorithm. By comparing our revised scheme's performance against the performance of the official MODIS cloud detection scheme, we conclude that the new scheme performs better than the earlier scheme which was not very good at thin cloud detection. 


\title{
Enhancing a Simple MODIS Cloud Mask Algorithm for the Landsat Data Continuity Mission
}

\author{
by \\ Michael J. Wilson ${ }^{1,2}$ and Lazaros Oreopoulos ${ }^{2}$ \\ 1. Joint Center for Earth Systems Technology, University of Maryland \\ Baltimore County, Baltimore, MD 21250 \\ 2. Climate and Radiation Laboratory, NASA-Goddard Space Flight Center, \\ Code 613, Greenbelt, MD 20771
}

Submitted to

IEEE Transactions on Geoscience and Remote Sensing

September 2011

Corresponding author address:
Lazaros Oreopoulos
NASA-GSFC
Code 613.2
Greenbelt, MD 20771
USA
Lazaros.Oreopoulos@onasa.gov 


\begin{abstract}
The upcoming Landsat Data Continuity Mission (LDCM) will include new channels centered around $1.38 \mu \mathrm{m}$ and $12 \mu \mathrm{m}$. This work studies the potential impact of these new channels on LDCM's cloud detection capabilities by using MODIS data as a proxy. Thresholds for the $1.38 \mu \mathrm{m}$ band and the so-called "split window" technique (using the brightness temperature difference of bands centered at $11 \mu \mathrm{m}$ and 12 $\mu \mathrm{m}$ ) were derived using ECMWF ERA-40 reanalysis atmospheric profiles and a MODIS-band radiance simulator. These thresholds were incorporated into a previously published cloud mask scheme, and applied on low- and mid-latitude MODIS radiance data from two different days, six months apart. While the previous scheme yields agreement rates to the MODIS cloud mask just below $80 \%$, the addition of the $1.38 \mu \mathrm{m}$ and split window tests increases the agreement $7-9 \%$. Use of the earlier scheme can continue for cloud masking of historical Landsat images and for carrying consistent cloud detection into the future. The enhanced scheme of this paper on the other hand, with its improved masking of primarily high thin clouds, can be combined with other masking techniques for generating a reliable LDCM cloud mask product that can potentially include confidence indicators based on the degree of agreement among multiple cloud masks.
\end{abstract}




\section{Introduction}

Clouds in Landsat imagery frequently obscure land features, the mission's prime target of interest. To date, only rudimentary information about clouds in Landsat-7 scenes has been provided in the form of cloud "scores" (cloud fractions) for the entire scene and each of its four quadrants. These cloud scores come from the Automated Cloud Cover Assessment (ACCA) algorithm [1]. The ACCA cloud scores help users screen for scenes with an overall low degree of cloud obscuration and are used in the long-term acquisition plan algorithm of the mission to determine whether a scene not in the contiguous USA should be acquired at the next available opportunity [2]. ACCA has the capability to provide a pixel-level cloud mask (e.g., [3]), but it has not been routinely used in that capacity.

The upcoming Landsat Data Continuity Mission (LDCM, http://ldcm.usgs.gov), on the other hand, seeks to develop a pixel-level cloud mask product. LDCM's Operational Land Imager (OLI) and Thermal InfraRed Sensor (TIRS) instruments in addition to bands similar to those on the current Landsat-5 and Landsat- 7 missions, will also have extra channels centered at $0.443 \mu \mathrm{m}$ and $1.38 \mu \mathrm{m}(\mathrm{OLI})$, and a second thermal infrared channel centered at $12 \mu \mathrm{m}$ (TIRS). The $1.38 \mu \mathrm{m}$ band and the two thermal bands are expected to enhance the ability of the LDCM to detect high clouds $([4] ;[5])$.

This paper presents a cloud masking scheme appropriate for the LDCM that improves upon a previous scheme applicable historical Landsat data by taking advantage of the new channels. The cloud detection scheme to be enhanced is a 
modification of the scheme first introduced by [6] (hereafter the "original" LTK scheme) as described in [3] (hereafter the "modified" LTK scheme). The original LTK scheme is a decision-tree algorithm that uses only visible and shortwave nearinfrared bands and was developed to operate on mid-latitude MODIS observations over land. With similar bands being present on the Thematic Mapper (TM) of Landsat-5 and Enhanced Thematic Mapper Plus (ETM+) Landsat-7, [3] demonstrated that with minor modifications the algorithm exhibited significant skill in pixel classification (clear vs. cloudy) for a collection of manually-masked Landsat scenes. This work seeks to incorporate the $1.38 \mu \mathrm{m}$ and thermal channels into the modified LTK scheme to produce an "enhanced" LTK scheme. Since no Landsat data with these new bands are currently available to evaluate the algorithm, we use Moderate resolution Imaging Spectroradiometer (MODIS) data as a proxy. Moreover, in the absence of a MODIS manual cloud mask, we use the official MODIS cloud mask (MOD35 product) as the cloud "truth". The sections that follow describe the development of the enhanced algorithm, including the methods used to determine thresholds for the new spectral tests, and provide metrics that demonstrate its improved performance when applied to MODIS data.

\section{Background on Algorithms}

The original LTK scheme suggested by [6] takes advantage of known spectral variations of certain surface types to classify pixels using MODIS Bands 1,2,3, and 6 (620-670 nm, 841-876 nm, 459-479 nm, and 1628-1652 nm, respectively; see Table 1). The scheme applies a series of threshold tests to identify pixels as non-vegetated 
land, snow/ice, water bodies, or cloudy. Any pixels that do not pass the tests for these categories are classified as vegetated land. [3] recognized that similar bands are available in the Landsat missions and applied the algorithm to a collection of 156 manually-masked Landsat-7 scenes (a subset of 212 scenes used by [1]). They found that by employing three minor modifications, the algorithm's performance improved such that clear and cloudy pixels were correctly distinguished $\sim 93 \%$ of the time. This level of agreement compared favorably to ACCA which includes thermal IR tests in addition to tests on various combinations of ETM+ solar reflectances. The modified LTK algorithm is shown in the unshaded part of Figure 1 using LDCM OLI band designations; Table 1 shows the correspondence between MODIS, Landsat-7 ETM+ and LDCM OLI/TIRS bands.

With the additional bands on the LDCM, further improvements can be introduced to the LTK scheme, especially for thin cloud detection, a weakness of the scheme identified by [3]. The $1.38 \mu \mathrm{m}$ band was designed for the detection of high clouds such as cirrus ([4]; [7]). Because of the strong water vapor absorption at this wavelength, high clouds, for which the above-cloud two-way water vapor path is small, appear relatively bright in $1.38 \mu \mathrm{m}$ imagery. Bright surfaces in very dry environments (e.g., polar regions) can also be frequently distinguished and can therefore be misidentified as clouds, but in general low clouds and surfaces in most environments appear dark (i.e., they contribute near-zero to the top of the atmosphere $1.38 \mu \mathrm{m}$ reflectance). We will describe later in this paper our own method of determination of a single global threshold for distinguishing between clear and high thin cloud pixels with the $1.38 \mu \mathrm{m}$ band. In addition to this band, 
LDCM allows the implementation of another technique that is effective in thin cloud and cloud edge detection, the so-called "split window" technique ([5]; [8]) Ice clouds of low to moderate optical thickness absorb and scatter less at $11 \mu \mathrm{m}$ than at $12 \mu \mathrm{m}$, so their transmittances are higher at $11 \mu \mathrm{m}$; this yields higher brightness temperatures at $11 \mu \mathrm{m}(\mathrm{T} 11)$ than at $12 \mu \mathrm{m}$ (T12), with the amount of the T11-T12 difference ("split") also affected by the amount of column water vapor. In this paper, as we will show later, split window differences are assigned thresholds that are dependent upon the $11 \mu \mathrm{m}$ brightness temperature, i.e., the technique is applied in a bispectral fashion.

The $1.38 \mu \mathrm{m}$ threshold and the split window technique are used here to improve upon the modified LTK scheme by reclassifying as cloudy a subset of the pixels originally classified as clear. The decision tree used to reclassify these pixels is shown in the shaded region of Figure 1 and its derivation will be further elaborated below. The entire algorithm of Figure 1 is referred to as the "enhanced" LTK scheme.

\section{MODIS Band Simulations}

The specific thresholds for the $1.38 \mu \mathrm{m}$ and split window tests of the enhanced LTK were determined with the aid of a special version of the Discrete Ordinates Radiative Transfer (DISORT) code described by [9] and [10] applied on atmospheric profiles obtained from European Center for Medium-Range Weather Forecasts (ECMWF) ERA 40 reanalysis data. The RT code was designed to simulate MODIS 
radiances, by including a package to calculate molecular absorption optical depths according to the correlated-k distribution (CKD) implementation of [11]. A single "snapshot" of ECMWF ERA-40 reanalysis data at $2.5^{\circ}$ resolution contains over ten thousand profiles that encompass a wide variety of horizontal and vertical water vapor and temperature variations over the earth's climate zones. We arbitrarily chose the data for January 15,2002 at $1200 \mathrm{Z}$. This data included atmospheric temperature, pressure, moisture, and geometrical height for 23 vertical levels. Because in an equal-angle grid high latitude grid boxes get a weighting disproportional to the area they occupy, a filter akin to that applied by the International Satellite Cloud Climatology Project (ISCCP, http://isccp.giss.nasa.gov/docs/mapgridinfo.html) was applied to create 6454 $(280 \mathrm{~km})^{2}$ equal-area grid boxes. The 6454 profiles were used to create clear-sky simulations of reflectances for MODIS bands $1,2,3,6$, and 26 (LDCM bands 4, 5, 2, 6, and 9, respectively; see Table I) and brightness temperatures for MODIS bands 31 and 32 (LDCM bands 10 and 11, respectively).

Cloudy-sky profiles were also generated for a variety of ice particle sizes and concentrations at different levels in the atmosphere. Clouds were place in the atmosphere with top pressures of $100 \mathrm{mb}, 150 \mathrm{mb}, 200 \mathrm{mb}, 250 \mathrm{mb}, 300 \mathrm{mb}$, and $400 \mathrm{mb}$, corresponding to discrete levels in the ECMWF ERA-40 reanalysis data. The clouds had pressure thicknesses corresponding to the thickness of the atmospheric layer directly beneath the cloud. For pressure tops between $100 \mathrm{mb}$ and $250 \mathrm{mb}$, clouds occupied a layer $50 \mathrm{mb}$ thick. For pressure tops at $300 \mathrm{mb}$ or $400 \mathrm{mb}$, clouds occupied a layer $100 \mathrm{mb}$ thick. The clouds were assigned optical 
depths of $0.1,0.3,0.5,0.75,1,2,3$, and 4 , and particles with effective ice diameters of 40, 70, and $100 \mu \mathrm{m}$. Each cloud top pressure, optical depth, and effective ice diameter combination was generated for each of the 6454 profiles, with only one cloud layer present at a time. The resulting profiles were then inserted in the DISORT algorithm. For the $1.38 \mu \mathrm{m}$ band, the solar zenith angle was fixed at 30 degrees (tests showed that in most cases the reflectance sensitivity was small for the typical range $30^{\circ}-60^{\circ}$ of LDCM), and the surface albedo was fixed at 0.25 , a value close to that expected for a wide range of non-ice land surfaces. For the thermal band simulations, the surface emissivity was set to 1 for both the 11 and $12 \mu \mathrm{m}$ bands. The phase functions for the assumed particle size distributions were provided internally by the RT simulation tool.

Extensive testing showed that to determine an appropriate $1.38 \mu \mathrm{m}$ threshold it was best to use the simulated reflectances for cloud optical thicknesses of $1,2,3$, and 4 . The desired threshold was identified by finding the reflectance corresponding to the lowest $1 \%$ of reflectances of simulated reflectances. The threshold reflectance derived this way corresponded to a value of 0.0113 . By using this threshold, only $1 \%$ of our cloudy simulations are misclassified as clear. When the threshold was applied to our separate subset of clear-sky simulations, approximately $93.7 \%$ of the clear simulations were below the threshold and therefore classified correctly. Had we included the simulations with optical depths less than 1, a lower threshold would have been needed to capture $99 \%$ of the cloudy profiles. This would have resulted in more incorrectly classified clear profiles. To keep the number of correctly classified clear profiles above $90 \%$ we would then have had to relax the 
requirement of only $1 \%$ cloud misclassifications. Also, note that over oceans where the surface reflectance is lower than 0.25 , the threshold determined with the above method may result in some missed high thin clouds.

To determine the threshold of the split window technique only clear-sky simulations of $11 \mu \mathrm{m}$ and $12 \mu \mathrm{m}$ brightness temperature were used. The values of the (T11, T11-T12) pairs for our clear-sky simulations are shown as gray points in Figure 2. Cloudy simulations generally exhibit larger values of T11-T12 for the same T11, so the T11-dependent threshold of T11-T12 can be determined by finding the upper envelope of the "cloud" of gray points. In practice, this was done as follows: The T11 brightness temperatures were sorted into $1 \mathrm{~K}$ bins. The largest T11-T12 difference in each bin was assigned as the bin threshold. If this threshold fell below 0.05 , then the threshold was set to 0.05 . In addition, the curve was smoothed such that a bin's threshold was always equal or greater than the threshold of the preceding bin. This resulted in a monotonically increasing curve beginning at 0.05 and rising to a maximum near 2.8, as shown in Figure 2. Any pair (T11, T11T12) below this curve is considered clear and any pair above the curve is considered cloudy. The thresholds represented by this curve capture $\sim 99 \%$ of our clear-sky simulations correctly. When applied to the cloudy simulations, $\sim 94 \%$ of profiles with clouds of optical thicknesses greater or equal to 0.5 were also classified correctly. The percentage of correctly classified profiles increases as clouds get thicker: for an optical depth of $3,99.4 \%$ of clouds are correctly classified, while only $80 \%$ of clouds are correctly classified for an optical depth of 0.5 . The skill of the detection with the above thresholds also improves as the cloud top pressure 
decreases (i.e., higher clouds). A similar curve is used for the split-window test of the operational MODIS mask algorithm [12], but our tests showed that our own curve works better in the context of the enhanced LTK algorithm. Finally, we found that when the above threshold selection methodology was applied to the ensemble of atmospheric profiles from a different date (July 15) of the ECMWF ERA-40 reanalysis data, the thresholds that resulted were very close to the ones found from the January profiles.

\section{Deriving the enhanced LTK scheme with the aid of MODIS data}

The thresholds determined through radiative simulations on the ECMWF ERA-40 reanalysis data were applied to the MODIS MOD021KM product, which contains radiances at $1 \mathrm{~km}$ resolution that can be converted to reflectances and brightness temperatures. The study used only MODIS-Terra data since the morning orbit closely corresponds to that of the current and future Landsat missions. The study was also restricted to data points equatorward of $60^{\circ}$ because of the inappropriateness of the LTK scheme for polar regions and the large decrease in the number of daytime cloud retrievals due to lack of illumination in the winter (northern) hemisphere. For the more challenging cloud detection locations poleward of $60^{\circ}$, alternative cloud masking techniques should be used. In total, 108 granules during January 15 th, 2002 contained daylight pixels with data points equatorward of $60^{\circ}$. 
The enhancement of the LTK scheme with the aid of the additional LDCM bands, indicated by the shaded region in Figure 1, was determined as follows: The modified LTK algorithm was used to classify the MODIS pixels into non-vegetated land, snow/ice, water bodies, vegetated land, or clouds, as usual. Each pixel was then retested using the split window and $1.38 \mu \mathrm{m}$ thresholds. Clear LTK pixels were changed to cloudy for four different reclassification scenarios: 1) the split window designated the pixel cloudy, 2) the $1.38 \mu \mathrm{m}$ threshold designated the pixel cloudy, 3 ) both designated the pixel cloudy, and 4) either algorithm designated the pixel cloudy. Similarly, LTK cloudy pixels were changed to clear following the same logic when the split window and/or the $1.38 \mu \mathrm{m}$ technique called a pixel clear. The results of each of these LTK enhancement scenarios were then compared against the MODIS cloud mask. The MOD35 product provides an overall cloud mask called the Unobstructed FOV Confidence Flag that categorizes each $1 \mathrm{~km}$ pixel as cloudy, uncertain/probably cloudy, probably clear, and confidently clear [12]. The first two categories, cloudy and uncertain/probably cloudy, were combined as a single cloudy category, while the probably clear and confidently clear categories were combined into a single clear category. It is this binary clear/cloud mask that provided the truth mask against the various LTK scenarios were tested. The best improvement in the modified LTK algorithm occurred over water bodies when either the split window or the $1.38 \mu \mathrm{m}$ technique called a pixel cloudy (an improvement of $7.03 \%$ ). Additional improvements occurred when either algorithm reclassified snow/ice pixels as cloudy (an improvement of 1.05\%) and when both algorithms reclassified non-vegetated land pixels as cloudy (an improvement of $0.18 \%$ ). These additional 
tests did not improve LTK agreement with MOD35 for vegetated land pixels or cloudy pixels. This means that more vegetated pixels were incorrectly classified as clouds than misclassified clear pixels corrected to cloudy.

Figure 3 contrasts maps of cloud fraction aggregated into 1 degree grid boxes for MOD35 algorithm and the enhanced LTK for the $60^{\circ} \mathrm{N}$ to $60^{\circ} \mathrm{S}$ latitude zone on January 15, 2002. The enhanced LTK shows the same pattern as MOD35, but tends to underpredict cloud cover, especially in the Atlantic Ocean and Pacific Ocean between $10^{\circ}$ and $30^{\circ} \mathrm{N}$ and the Eastern Pacific Ocean near the equator. Figure 4 compares the zonally-averaged cloud fraction derived from the enhanced LTK and MOD35, and also shows the improvement the additional tests bring to the modified LTK of [3] by also displaying the modified LTK zonal cloud fraction. Since the enhanced LTK does not reclassify cloudy pixels as clear, the enhanced LTK is always at least as cloudy as the modified LTK. The largest differences in cloud cover between the modified LTK and the enhanced LTK exist between $20^{\circ} \mathrm{S}$ and $10^{\circ} \mathrm{N}$, where tropical cirrus clouds are most prevalent. The largest differences between the enhanced LTK and MOD35 exist between $5^{\circ} \mathrm{S}$ and $25^{\circ} \mathrm{N}$, mainly in the regions highlighted previously in Figure 3. The mid-latitudes in both hemispheres tend to generally show fair agreement in cloud fraction between MOD35, the enhanced LTK, and the modified LTK. The domain-average cloud fractions for this latitude zone are $68.7 \%$ for MOD35, 59.5\% for enhanced LTK and $48.6 \%$ for modified LTK. These results indicate that with a more limited arsenal of spectral tests, the enhanced LTK will inevitably miss a substantial fraction of cloudy pixels detected by the most 
sophisticated MODIS cloud mask, but will nonetheless improve significantly the performance of the previous version of LTK.

The MOD06 product includes retrievals of cloud top pressures at a $5 \mathrm{~km}$ resolution. This product was used to classify any MOD35 cloudy pixels falling within a MOD06 cloud top pressure retrieval into three categories: high clouds (with cloud tops < $400 \mathrm{mb}$ ), mid-level clouds (with tops $\geq 400 \mathrm{mb}$ and $<850 \mathrm{mb}$ ), and low clouds (with cloud tops $\geq 850 \mathrm{mb}$ ). Table II shows the performance of the modified LTK and enhanced LTK schemes relative to the MOD35 cloud mask product. Cloud detection performance is also broken by cloud height category as determined by the MOD06 cloud pressure product. The split window and $1.38 \mu \mathrm{m}$ tests improve the overall performance of the enhanced LTK over the modified LTK by nearly $8.7 \%$. Correct cloud detection improves by nearly 15\% in the enhanced LTK, while clear pixel detection only decreases by about $4.4 \%$. The most dramatic improvements in cloud detection occur for high clouds (24\%), while mid-level clouds improve by about $7 \%$ and low clouds improve by about $16 \%$. The enhanced LTK captures over $95 \%$ of the high and mid-level clouds detected by MODIS. However, low clouds are still a weakness of the algorithm, with only $\sim 60 \%$ of low level clouds correctly classified after the application of the enhanced LTK.

The robustness of the above performance results was tested with a second set of data from MODIS-Terra on July 15, 2002. In all, 104 granules contained sunlit data equatorward of $60^{\circ}$ on July 15 . The modified LTK algorithm of [3] correctly classified $78.1 \%$ of the data in July, while the enhanced LTK classified $85.2 \%$ of the 
data correctly. The similarity of the overall performance between July and January is consistent with the aforementioned proximity of the spectral thresholds derived from the July ECMWF reanalysis data. Moreover, the July performance of enhanced LTK suggests that the cloud properties between $60^{\circ} \mathrm{N}$ and $60^{\circ} \mathrm{S}$ are collectively alike between the two months.

\section{MODIS Granule Analysis}

The performance of the modified LTK and enhanced LTK algorithms was also examined at the level of individual granules (MODIS scenes). An example for a select granule is shown in Figure 5. As expected by the results from Table II, the modified LTK algorithm struggles with the high thin clouds in the upper part of the image, while the enhanced LTK captures a significant number of these clouds.

The original 108 granules were filtered to only include the 97 granules with average latitudes below $60^{\circ}$. The percentage agreement at the pixel level for the modified and enhanced LTK with respect to MOD35 for these 97 granules is shown in Figure 6. The original LTK has an overall agreement of $79.2 \%$ per scene, while the enhanced LTK has an overall agreement of $87.7 \%$ per scene (Table II), which is an improvement of 8.5\%. The enhanced LTK improves the MOD35 agreement for 92 granules and worsens the agreement for only 5 granules. Using $80 \%$ agreement as the boundary between good and weak performance, the modified LTK meets or exceeds this baseline for 51 of the 97 granules, while the enhanced LTK meet sor exceeds this baseline for 83 of the 97 granules. 
The percentage agreement with respect to MOD35 is plotted in Figure 7 versus the absolute granule cloud fraction errors for both the modified and enhanced LTK. When the scene cloud fraction error is examined against the percentage level of agreement at the pixel level, the degree of cancellation of clear and cloudy detection errors is identified. To understand this figure, consider as an extreme example a granule to which both the LTK and MOD35 assign a cloud fraction of 50\% 0.5 if cloud fraction is measured in the scale from 0 to 1 ). However, if the LTK and MOD35 schemes are exactly opposite in the pixels they classify as cloudy their level of agreement would be $0 \%$, even if the absolute scene cloud fraction error is also $0 \%$ (since the scenes are both $50 \%$ cloudy). This occurs because the misclassifications of clear and cloudy pixels cancel each other out. One ideally pursues a $0 \%$ cloud fraction error that corresponds to $100 \%$ pixel-level agreement. Figure 7 shows deviations from this ideal. The $80 \%$ agreement and the $20 \%$ absolute scene cloud fraction error lines were placed in Figure 7 to create a quadrant that contains these granules with significant cancellation errors, i.e., lower-left quadrant granules have low pixel-level agreement, but similar cloud fractions to MOD35. The original LTK has 10 granules in this quadrant, while the enhanced LTK has 6 granules in this quadrant. This suggests that while cancellation errors are not very common in either the modified LTK or the enhanced LTK, the new scheme is once again better with regard to this performance metric. 


\section{Conclusions}

The work described here illustrates the importance for cloud detection of the availability of the $1.38 \mu \mathrm{m}$ cirrus band and of two thermal bands centered at 11 and $12 \mu \mathrm{m}$ for the future LDCM mission. These bands are expected to aid in the detection of high and thin clouds in LDCM imagery. Their potential impact is demonstrated through the use of MODIS-Terra data. MODIS has the above bands, as well as the bands required to apply the Landsat cloud mask (modified LTK scheme) discussed in [3]. MODIS data are therefore suitable for use as a testbed on which enhancements of the modified LTK scheme can be developed.

Thresholds for the $1.38 \mu \mathrm{m}$ channel and the split -window technique were derived by running a MODIS radiance simulation tool based on the DISORT radiative transfer code on ECMWF ERA-40 reanalysis data from January 15, 2002. Both the original clear-sky profiles and a multitude of our own profiles generated by inserting various ice clouds at the upper levels of the troposphere were used in these radiative transfer simulations. A single global threshold was derived for the $1.38 \mu \mathrm{m}$ channel, while split-window (brightness temperature differences between 11 and $12 \mu \mathrm{m}$ ) thresholds were calculated for $1 \mathrm{~K}$ bins of the $11 \mu \mathrm{m}$ brightness

temperature. These thresholds were then incorporated in an earlier cloud masking scheme and the scheme was subsequently applied on MODIS radiances from the same day, as well as MODIS data from six months later. All data was under daylight conditions and equatorward of $60^{\circ}$. The results for January showed an overall agreement with the official MODIS cloud mask (MOD35) of $79.2 \%$ for the modified 
LTK and an overall agreement of $87.8 \%$ for the new version of the LTK enhanced with the $1.38 \mu \mathrm{m}$ and split-window tests. The results for July showed an overall agreement of $78.1 \%$ for the modified LTK alone and an overall agreement of $85.2 \%$ for the enhanced LTK, very similar to the January dataset. This shows that the thresholds derived through the DISORT analysis are appropriate for MODIS and applicable to multiple days. When the performance of the modified LTK and the enhanced LTK were examined at the level of MODIS granules we found that for the 97 granules with an average latitude less than $60^{\circ}$, the modified LTK had at least $80 \%$ agreement with the MOD35 cloud mask for 51 of the 97 granules. The enhanced LTK had at least $80 \%$ agreement to MOD35 for 83 of the 97 granules.

Given the similarities between the MODIS and LDCM bands used in the enhanced LTK scheme, the cloud masking algorithm proposed here can be applied to the future LDCM mission. Whereas the modified LTK of [3] allows for cloud masking without the TIRS instrument and provides a technique that can produce cloud masks consistent with previous Landsat missions, the enhanced LTK provides an improved cloud masking technique that utilizes the new channels of LDCM and can be combined with an operational cloud mask for a future cloud mask product and to infer confidence levels in the operational cloud detection. While most of the improvement in the enhanced LTK compared to its previous incarnation comes indeed from cloud detection over water surfaces, which are often of secondary interest to Landsat missions, knowledge of cloud amounts contributed by the watercovered part of a scene is still important information for evaluating scene quality in many applications. Moreover, having the best possible cloud scores in commonly 
occuring scenes containing land-water mixtures will be very valuable in fine-tuning the implementation of the LDCM long-term acquisition plan.

\section{ACKNOWLEDGMENT}

Funding by USGS under contract 06CRCN0022 is gratefully acknowledged. 


\section{References}

[1] R. R. Irish, J. L. Barker, S. N. Goward, and T. Arvidson, "Characterization of the Landsat-7 ETM automated cloud-cover assessment (ACCA) algorithm,"

Photogramm. Eng. Remote Sens., vol. 72, no. 10, pp. 1179-1188, Oct. 2006.

[2] T. Arvidson, J. Gasch, and S. N. Goward, “Landsat 7's long term acquisition plan An innovative approach to building a global archive", Remote Sensing of Environment, vol. 78, no. 1-2, pp. 13-26, Oct. 2001.

[3] L. Oreopoulos, M. J. Wilson, T. Várnai, "Implementation on Landsat Data of a Simple Cloud-Mask Algorithm Developed for MODIS Land Bands," IEEE Geosci. And Remote Sens. Letters, Vol. 8, No. 4, p. 597-601, July 2011.

[4] B.-C. Gao and Y. J. Kaufman, "Selection of the 1.375-um MODIS Channel for Remote Sensing of Cirrus Clouds and Stratospheric Aerosols from Space," J. Atmos. Sci., 52, p. 4231-4237, 1995.

[5] T. Inoue, "A Cloud Type Classification with NOAA 7 Split-Window Measurements," J. Geophys. Res., Vol. 92, No. D4, p. 3991-4000, 1987.

[6] Y. Luo, A. P. Trishchenko, K. V. Khlopenkov, "Developing Clear-sky, Cloud, and Cloud Shadow Mask for Producing Clear-Sky Composites at 250-meter Spatial Resolution for the Seven MODIS Land Bands over Canada and North America," Remote Sens. Environ., vol. 112, no. 12, pp. 4167-4185, Dec. 2008.

[7] B.-C. Gao, P. Yang, W. Han, R.-R. Li, W. J. Wiscombe, "An Algorithm Using Visible and 1.38-um Channels to Retrieve Cirrus Cloud Reflectances From Aircraft and Satellite Data," IEEE Trans. on Geosci. and Remote Sens., Vol. 40, No. 8, 2002. 
[8] R. W. Saunders and K. T. Kriebel, "An Improved Method for Detecting Clear Sky and Cloudy Radiances from AVHRR Data," Int. J. Remote Sens., Vol. 9, Issue 1, p. 123 $150,1988$.

[9] S.-H. Ham, B.-J. Sohn, P. Yang, B. A. Baum, "Assessment of the Quality of MODIS Cloud Products from Radiance Simulations," J. Appl. Meteor. and Clim., Vol. 48, Issue 8, p. 1591-1612, 2009.

[10] B.-J. Sohn, S. Ham, P. Yang, and B. A. Baum, "Simulation of Fifteen MODIS Bands for the Assessment of MODIS Cloud Products," in Hyperspectral Imaging and Sensing of the Environment, OSA Technical Digest (CD), Optical Society of America, paper HWB3, available at http://www.opticsinfobase.org/abstract.cfm?URI=HISensE-2009-HWB3.

[11] D. P. Kratz, "The correlated k-distribution technique as applied to the AVHRR channels", J. Quant. Spectrosc. Radiat. Transfer, 53, 501-517, 1995.

[12] S. Ackerman, K. I. Strabala, W. P. Menzel, R. A. Frey, C. C. Moeller, L. E. Gumley, "Discriminating clear sky from clouds with MODIS," J. Geophys. Res., vol. 103, no. D24, pp. 32141-32157, 1998. 


\section{List of Tables}

Table I: Correspondence between MODIS, Landsat-7 ETM+, and LDCM OLI/TIRS

bands used in the various versions of the LTK cloud detection scheme.

TABLE I

CORRESPONDENCE BETWEEN MODIS, LANDSAT-7 ETM+, AND LDCM OLI/TIRS BANDS

\section{MODIS}

Landsat-7 ETM+

\section{LDCM OLI/TIRS}

\begin{tabular}{l|l|l} 
Band $3(459-479 \mathrm{~nm})$ & Band 1 $(450-515 \mathrm{~nm})$ & Band 2 $(450-515 \mathrm{~nm})$
\end{tabular}

\begin{tabular}{l|l|l}
\hline Band 1 $(620-670 \mathrm{~nm})$ & Band 3 $(630-690 \mathrm{~nm})$ & Band 4 $(630-680 \mathrm{~nm})$
\end{tabular}

\begin{tabular}{|l|l|l}
\hline Band 2 $(841-876 \mathrm{~nm})$ & Band 4 $(750-900 \mathrm{~nm})$ & Band 5 $(845-885 \mathrm{~nm})$
\end{tabular}

Band 6(1628-1652 nm) Band $5(1550-1750 \mathrm{~nm})$ Band 6 (1560-1660 nm)

Band 26 (1360-1390 nm) Band 9 (1360-1390 nm)

Band 31 (10780-11280 nm)

Band $6(10400-12500 \mathrm{~nm})$

Band $32(11770-12270 \mathrm{~nm})$

Band $10(10300-11300 \mathrm{~nm})$

Band $11(11500-12500 \mathrm{~nm})$ 
Table II: Performance of the modified and enhanced LTK relative to the MODIS MOD35 cloud mask. The performance is shown as percentage agreement for all cloudy pixels and for cloudy pixels classified with respect to their MOD06 cloud top pressure: less than $400 \mathrm{mb}$ (high clouds), between $400 \mathrm{mb}$ and $850 \mathrm{mb}$ (mid-level clouds), greater than $850 \mathrm{mb}$ (low clouds). The percentage agreement for MOD35 clear pixels is also provided. Overall performance is determined by weighting by the overall cloud fraction of $68.1 \%$. The cloud fraction breakdown is $23.1 \%$ high, $29 \%$ mid-level, and $16 \%$ low clouds.

TABLE II

PERFormanCE of THE Two FlaVors of THE LTK SCHEME

\begin{tabular}{|c|c|c|}
\hline & Modified LTK & Enhanced LTK \\
\hline All Clouds & $72.90 \%$ & $87.72 \%$ \\
\hline $\begin{array}{c}\text { High Clouds } \\
\text { (Cloud Top Pressure }<400 \mathrm{mb} \text { ) }\end{array}$ & $72.91 \%$ & $97.02 \%$ \\
\hline $\begin{array}{c}\text { Mid-Level Clouds } \\
\text { (Cloud Top Pressure }>=400 \mathrm{mb} \text { and }<850 \mathrm{mb})\end{array}$ & $89.45 \%$ & $96.31 \%$ \\
\hline $\begin{array}{c}\text { Low Clouds } \\
\text { (Cloud Top Pressure }>=850 \mathrm{mb} \text { ) }\end{array}$ & $45.29 \%$ & $61.56 \%$ \\
\hline Clear & $92.53 \%$ & $88.11 \%$ \\
\hline $\begin{array}{c}\text { Overall Performance } \\
\text { (MOD35 Data is 68.1\% Cloudy, 31.9\% Clear) }\end{array}$ & $79.16 \%$ & $87.84 \%$ \\
\hline
\end{tabular}




\section{Figure Captions}

Figure 1: The flow chart of the LTK pixel classification scheme. The unshaded region shows the LTK algorithm as modified by [3]. The shaded region shows the inclusion of the additional spectral tests to create the enhanced LTK.

Figure 2: Curve showing the brightness temperature difference T11-T12 separating clear from cloudy pixels as a function of $11 \mu \mathrm{m}$ brightness temperature T11. Values above this curve indicate cloudy pixels, while values below this curve indicate clear pixels. The cloud of gray points represents the values of (T11,T11-T12) pairs for all the 6454 clear-sky gridpoints of our simulation.

Figure 3: Cloud fraction on January 152002 from the MOD35 cloud masking algorithm (top) vs. cloud fraction from the enhanced LTK scheme. Both maps are gridded at a resolution of $1^{\circ}$ latitude by $1^{\circ}$ longitude. Only pixels between $60^{\circ} \mathrm{N}$ and $60^{\circ} \mathrm{S}$ are included in these images. However, the land/water mask is provided in the polar regions for reference.

Figure 4: Zonal (longitudinally-averaged) cloud fraction of $1^{\circ}$ resolution corresponding to Figure 4. The zonal cloud fraction from the modified LTK of [3] is also shown for reference.

Figure 5: Cloud masks for a MODIS-Terra granule off the coast of South Africa on January 15, 2002 at $0910 \mathrm{Z}$. The top image shows the MOD35 cloud mask classified by the MOD06 Cloud Top Pressure Product. Clouds are in blue, green, and red while clear skies are generated by a false-color 4-3-2 RGB image (near-infrared, red, and 
green respectively for RGB). The bottom images show the modified LTK cloud mask and the enhanced LTK cloud mask; clouds are in yellow.

Figure 6: Comparison between modified and enhanced LTK algorithm level of agreement with respect to the MODIS MOD35 cloud mask for the 97 MODIS granules of January 15, 2002. Pixels in the upper-right quadrant are granules with good agreement ( $>80 \%$ ) in both the original LTK and the enhanced LTK. Pixels in the lower-left quadrant have poor agreement $(<80 \%)$ for both algorithms. Pixels in the upper-left quadrant are granules that have poor agreement for the original LTK but have good agreement for the enhanced LTK. Pixels in the lower-right quadrant have good agreement for the original LTK but poor agreement for the enhanced LTK.

Figure 7: Scatterplots of percentage pixel-level agreement vs. granule absolute cloud fraction error for the modified and enhanced LTK algorithm. Each point represents a MODIS granule from January 15, 2002. Points that fall in the lower-left quadrant formed by the vertical and horizontal lines represent granules with a large cancellation error (absolute cloud fraction error $<20 \%$ ). Granules in this quadrant have low pixel-level agreement relative to MOD35 but granule cloud fractions that are close to MOD35. 


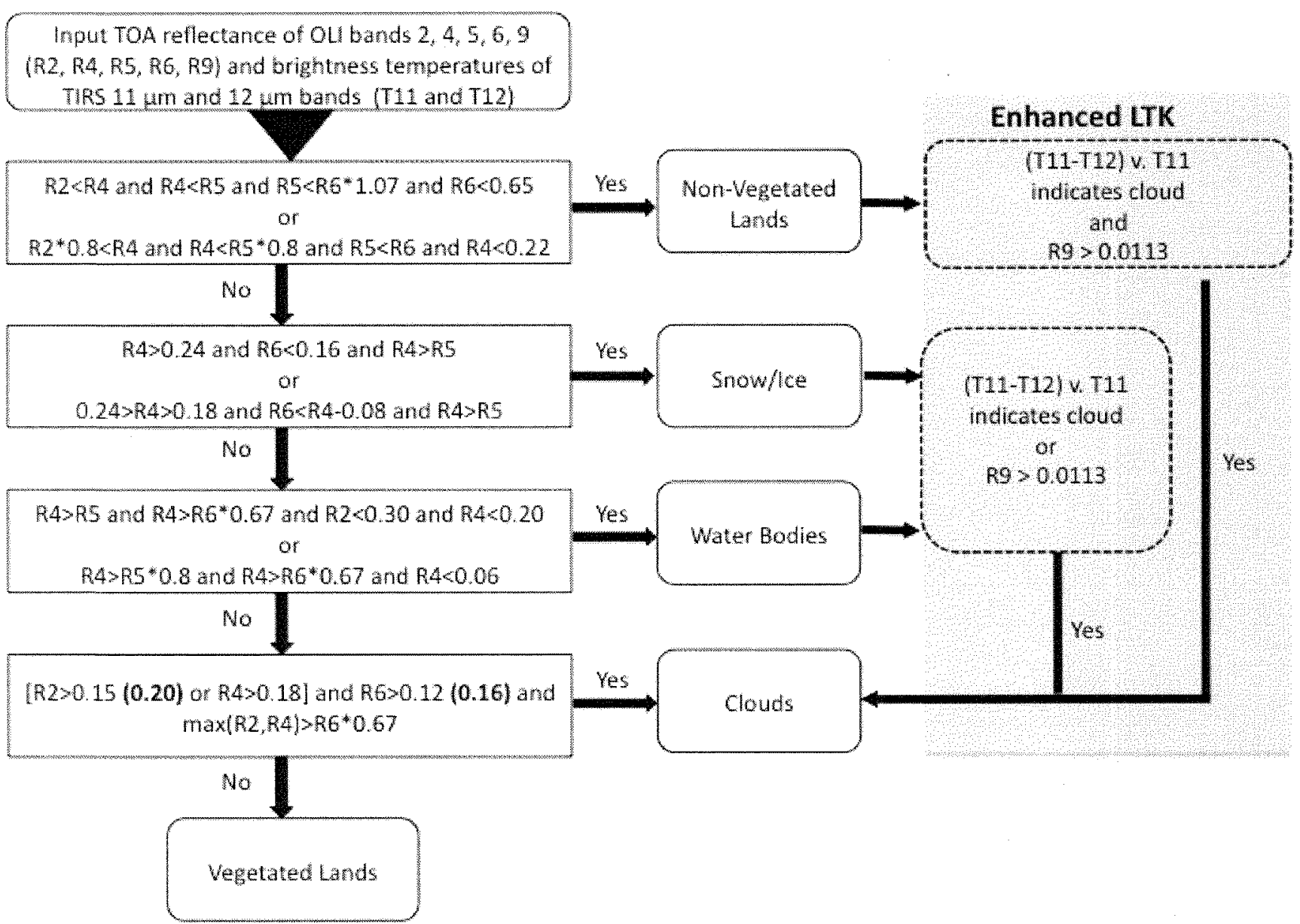

No enhancement for Vegetated Lands 


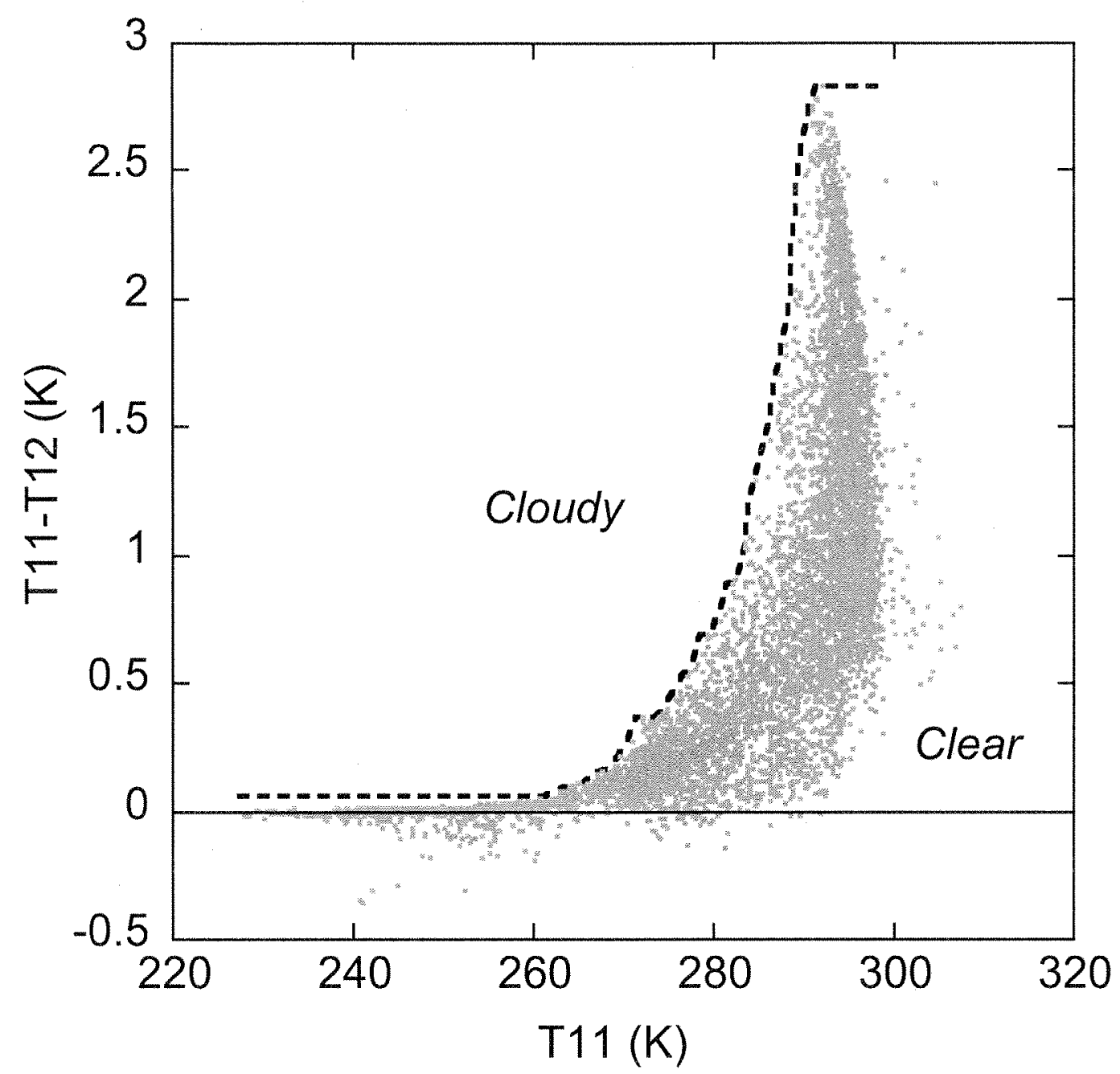

Figure 2 
MOD35 Cloud Fraction

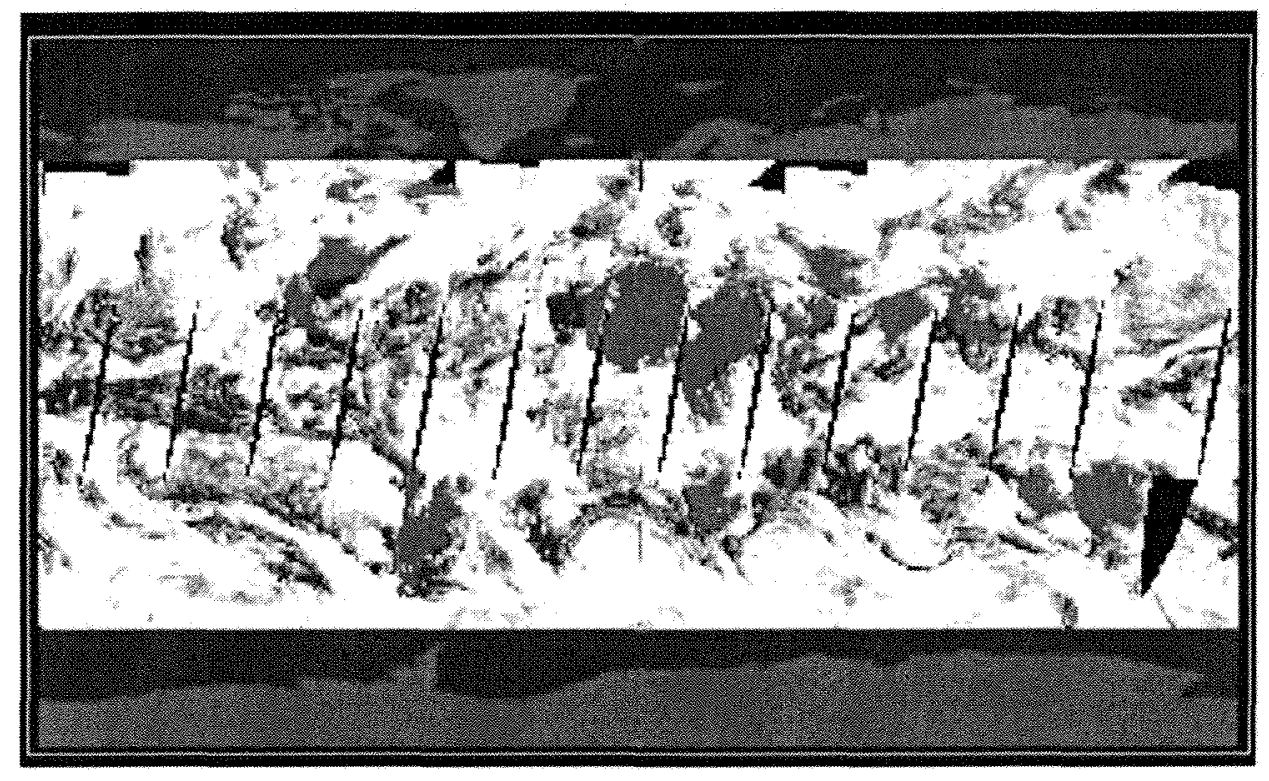

Enhanced LTK Cloud Fraction

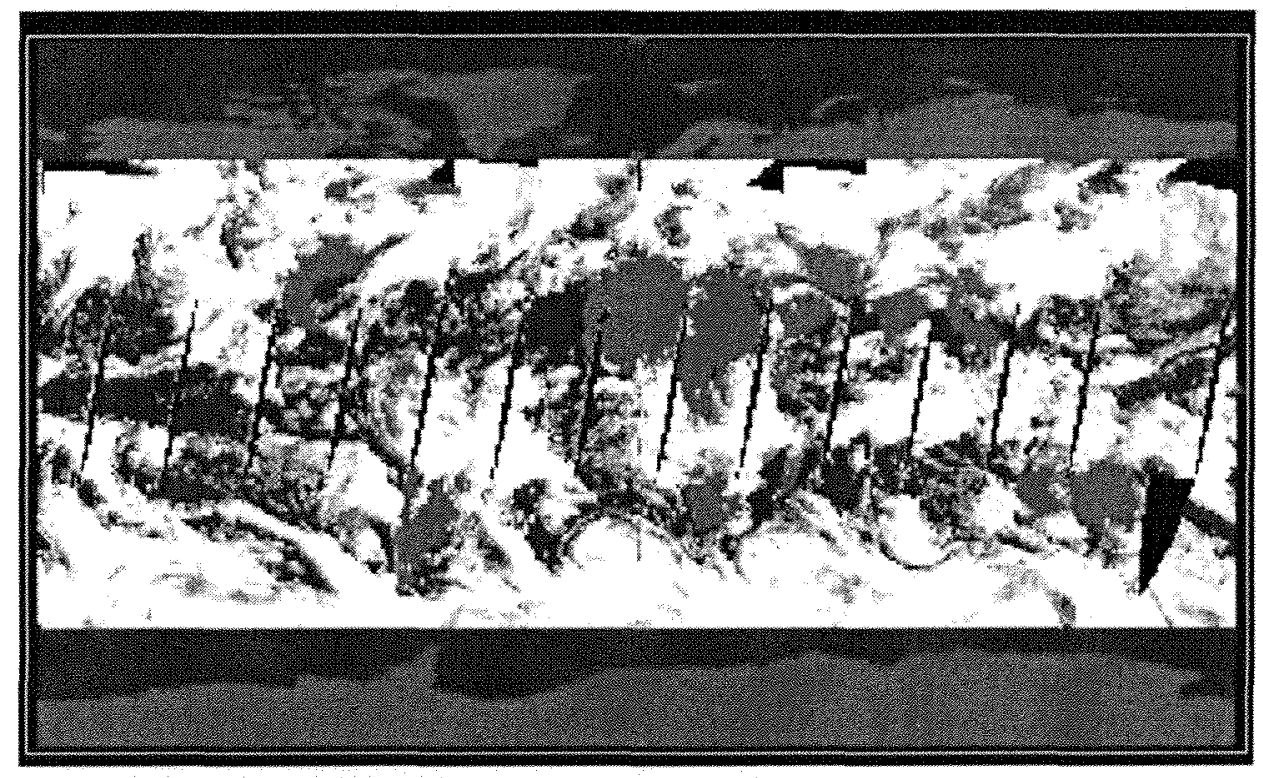

No
Retrieval

Figure 3 


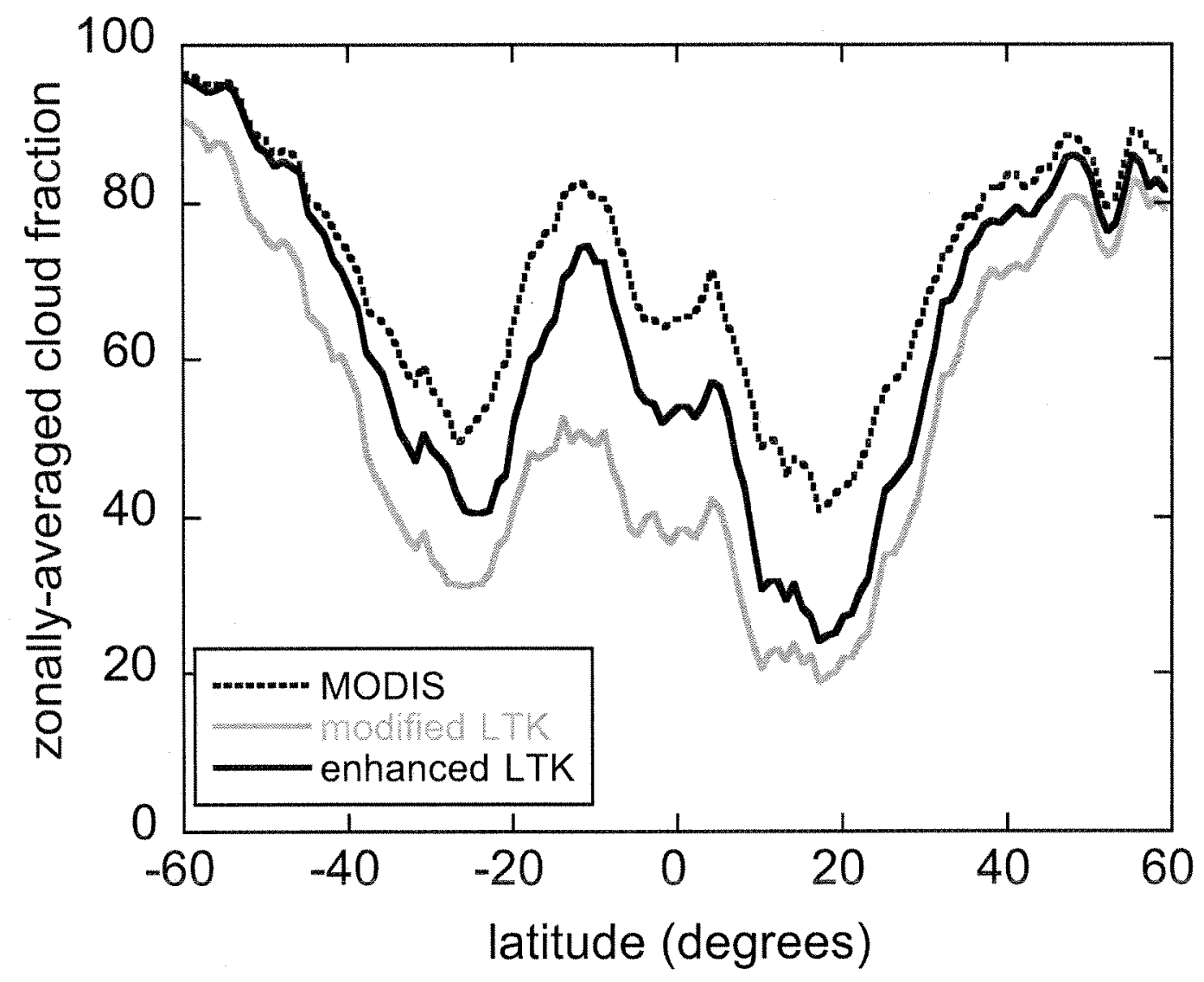

Figure 4 


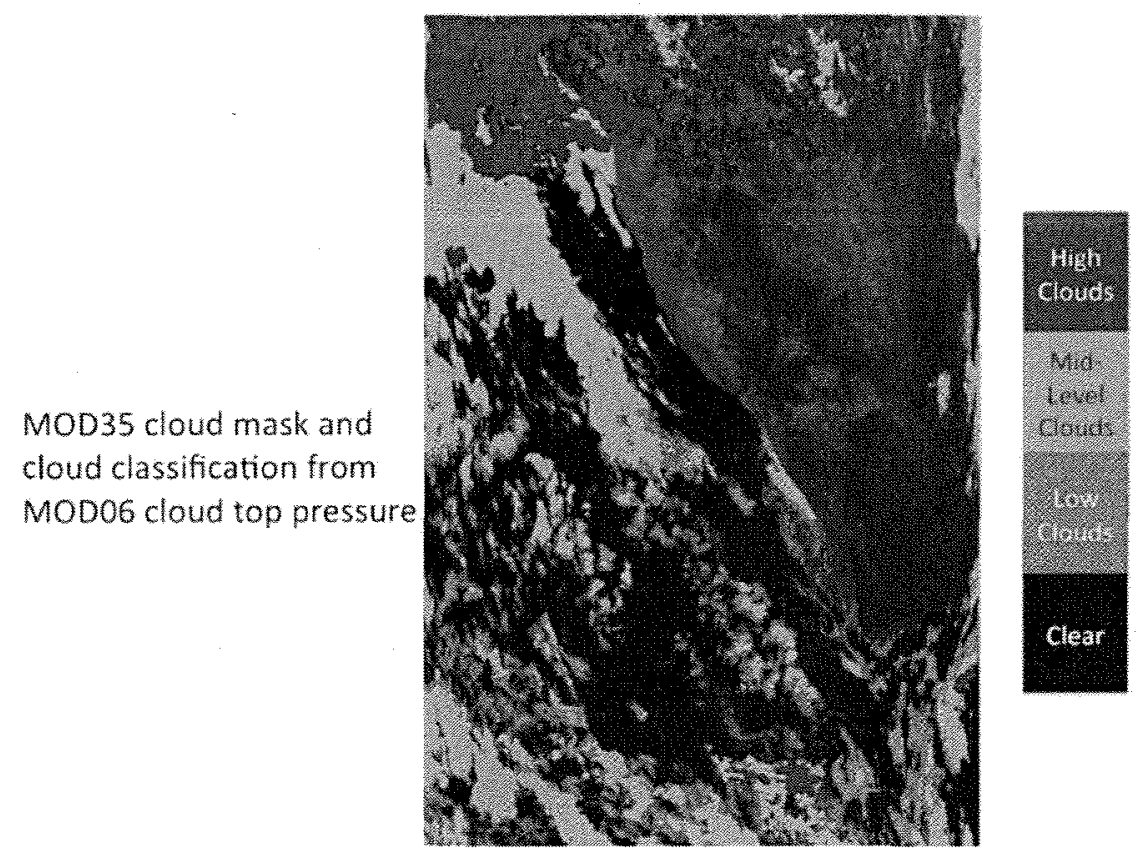

Modified LTK cloud mask

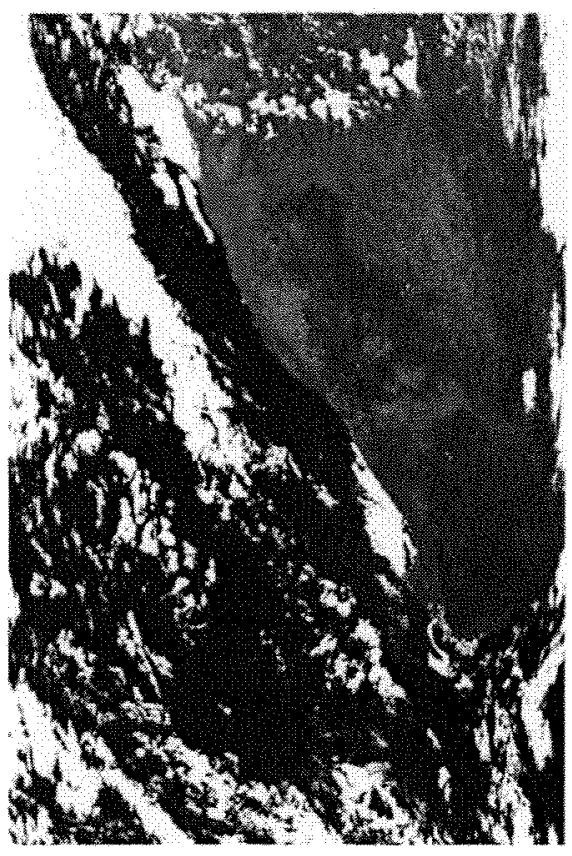

Enhanced LTK cloud mask

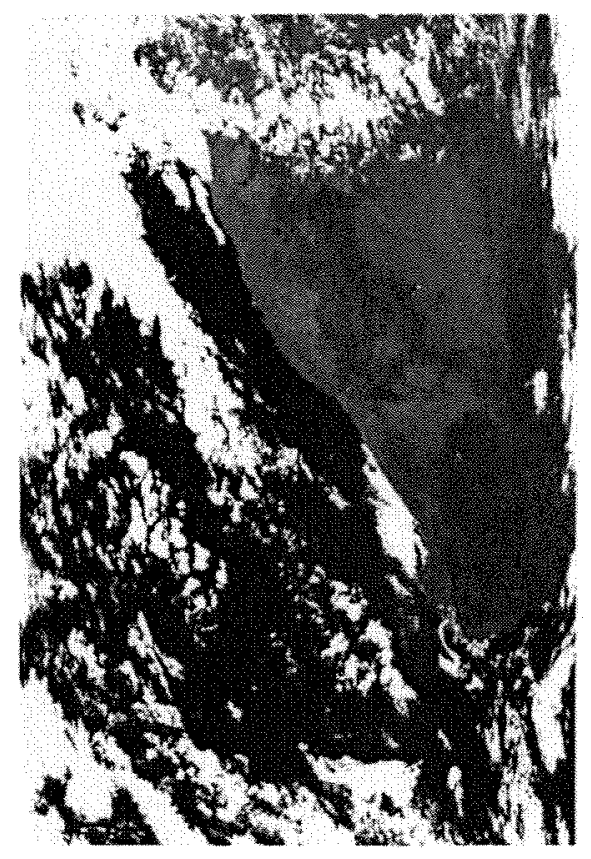

Figure 5 


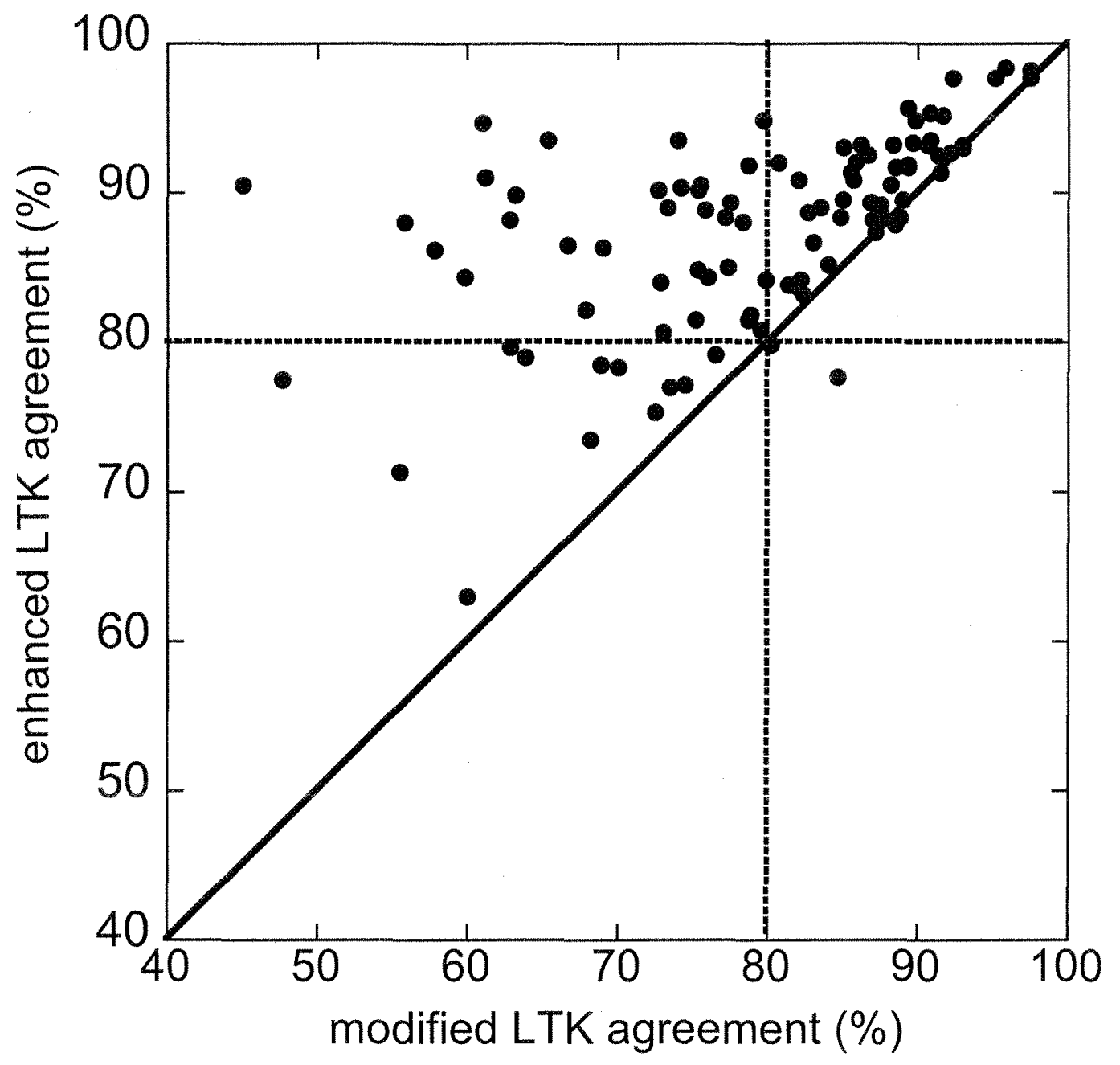

Figure 6 

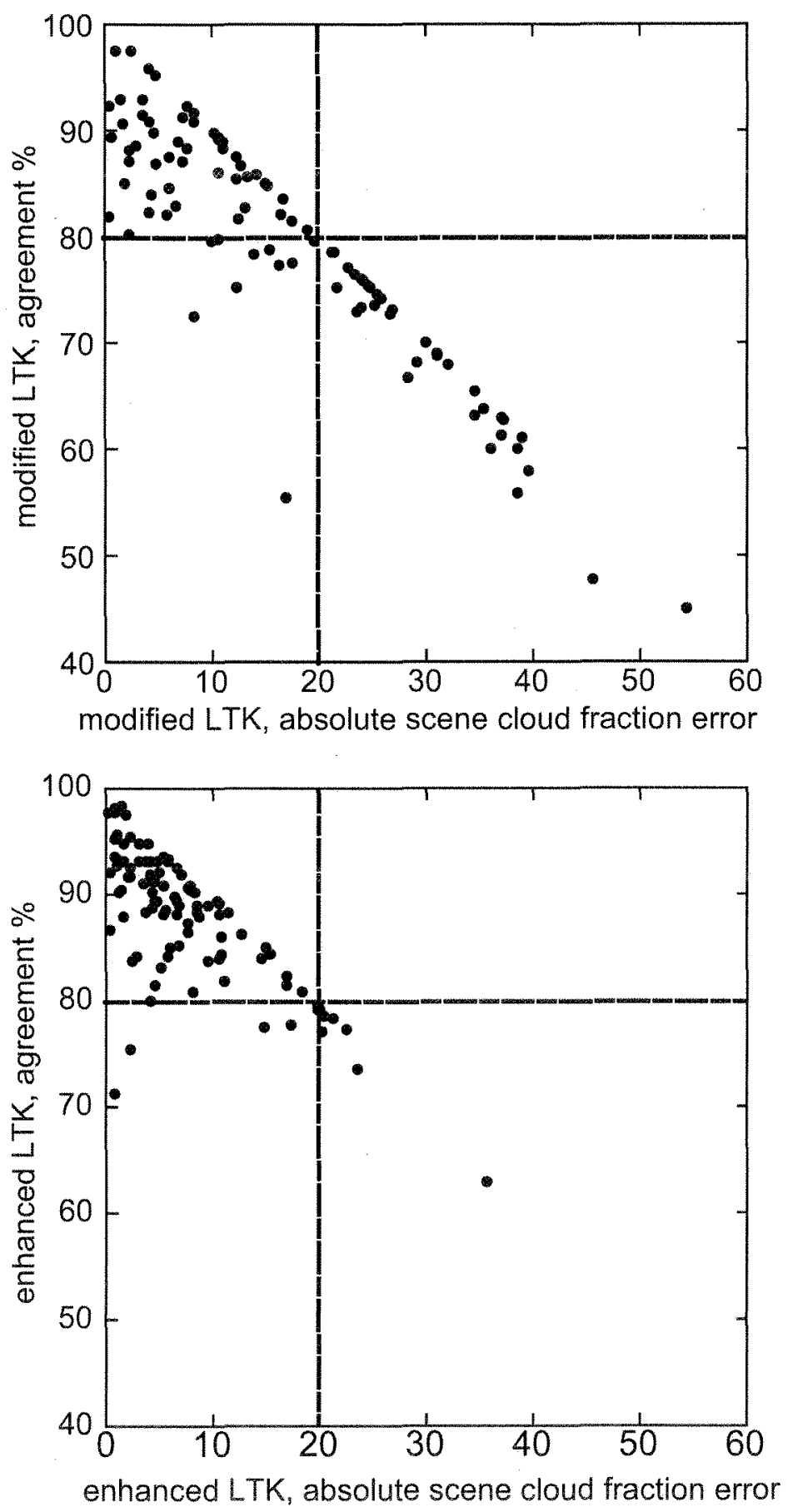

Figure 7 